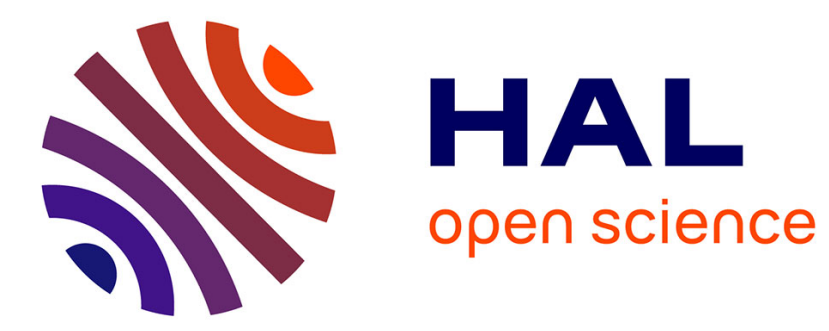

\title{
Role of adipose tissues in osteoarthritis
}

Natalia Zapata-Linares, Florent Eymard, Francis Berenbaum, Xavier Houard

\section{To cite this version:}

Natalia Zapata-Linares, Florent Eymard, Francis Berenbaum, Xavier Houard. Role of adipose tissues in osteoarthritis. Current Opinion in Rheumatology, 2021, 33 (1), pp.84-93. 10.1097/BOR.0000000000000763 . hal-03263622

\section{HAL Id: hal-03263622 \\ https: / hal.sorbonne-universite.fr/hal-03263622}

Submitted on 17 Jun 2021

HAL is a multi-disciplinary open access archive for the deposit and dissemination of scientific research documents, whether they are published or not. The documents may come from teaching and research institutions in France or abroad, or from public or private research centers.
L'archive ouverte pluridisciplinaire HAL, est destinée au dépôt et à la diffusion de documents scientifiques de niveau recherche, publiés ou non, émanant des établissements d'enseignement et de recherche français ou étrangers, des laboratoires publics ou privés. 


\section{Role of adipose tissues in osteoarthritis}

2

3 Natalia Zapata-Linares ${ }^{1}$, Florent Eymard ${ }^{2,3}$, Francis Berenbaum ${ }^{1,4,}{ }^{*}$ and Xavier Houard ${ }^{1}$

4

$5{ }^{1}$ Sorbonne Université, INSERM, Centre de Recherche Saint-Antoine (CRSA), F-75012 Paris,

6 France.

$7 \quad{ }^{2}$ Department of Rheumatology, AP-HP Henri Mondor Hospital, F-94010, Créteil Cedex, France.

$8{ }^{3}$ Gly-CRRET Research Unit 4397, Université Paris-Est Créteil, F-94010, Créteil, France.

$9{ }^{4}$ Sorbonne Université, INSERM CRSA, AP-HP Hopital Saint Antoine, Paris, 10

11

12 * Address for correspondence:

13 Francis Berenbaum, MD, PhD.

14 Centre de Recherche Saint-Antoine,

15184 rue du Faubourg Saint-Antoine

16 F-75012 Paris

17 France

18 Email: francis.berenbaum@aphp.fr

19

20 Word count: 2506

21

22 
Purpose of the review. Epidemiologic studies reveal that the link between obesity and 26 osteoarthritis (OA) cannot be uniquely explained by overweight-associated mechanical overload.

27 For this reason, much attention focuses on the endocrine activity of adipose tissues. In addition

28 to the systemic role of visceral and subcutaneous adipose tissues, many arguments highlight the 29 involvement of local adipose tissues in OA.

30 Recent findings. Alteration in magnetic resonance imaging signal intensity of the 31 infrapatellar fat pad may predict both accelerated knee OA and joint replacement. In this context, 32 recent studies show that mesenchymal stromal cells could play a pivotal role in the pathological 33 remodeling of intra-articular adipose tissues in OA. In parallel, recent findings underline bone 34 marrow adipose tissue as a major player in the control of the bone microenvironment, suggesting 35 its possible role in OA.

36 Summary. The recent description of AT of various phenotypes within an osteoarthritic joint

37 allows us to evoke their direct involvement in the initiation and progression of the osteoarthritic 38 process. We can expect in the near future the discovery of novel molecules targeting these 39 tissues.

41 Keywords: osteoarthritis, adipose tissue, adipokines, intra-articular adipose tissues, bone 42 marrow adipose tissue 


\section{Introduction}

Osteoarthritis (OA) is the most common musculoskeletal disease and is one of the leading

47 causes of disability worldwide. The disability-adjusted life years (DALYs) index for OA rose by

$48 \quad 34.8 \%$ between 2005 and 2015 [1]. The increase in the number of OA patients cannot be

49 explained solely by the ageing of the world population, highlighting the importance of other risk factors. Obesity is the main modifiable risk factor for OA [2]. The World Health Organization

51 estimates that the worldwide prevalence of obesity nearly tripled since 1975 with more than 1.9

52 billion adults overweight in 2016, among them 650 million were obese.

The role of overweight-associated mechanical overload has long been pointed out to explain

54 the link between OA and obesity. Clinical studies indeed described positive correlations between

55 body mass index (BMI) and both the incidence and the progression of knee OA [3, 4]. However,

56 obesity also impacts non-weight bearing joints [5], suggesting that factors other than mechanical

57 overload also contribute to joint damage in obese patients.

58 In addition to their role in energetic metabolism, adipose tissues (AT) are endocrine organs

59 releasing factors acting on distant organs. These factors, of which the prototype and the better

60 known is leptin, are defined as adipokines [6]. Blood levels of leptin increase with BMI as they

61 are in OA patients [7, 8]. Evidence argue for a role of leptin in OA [9]. Numerous other

62 adipokines are produced by AT and their secretion pattern is also affected by obesity [10]. This

63 altered secretion pattern of AT related to obesity reflects modifications in their tissue

64 composition as well as modifications in the phenotype of cells present within AT.

65 AT do not constitute a unique entity. White and brown AT have been described, differing by 66 their developmental origin, the phenotype of their adipocytes and their function in energetic 
67 metabolism and thermogenesis. Moreover, multiple white AT (WAT) exist, present in the whole

68 body as separate fat pads with specific features. In this review, we will describe the known

69 features of different AT, including subcutaneous, visceral, intra-articular and bone marrow AT, 70 and will focus on their potential roles in OA.

71

72

Methodology

73

74 A search for original articles published between January 2017 and October 2020 was 75 performed on PubMed. The search terms used were "Adipose tissue AND Osteoarthritis" for 76 reviews, "Adipokines AND Osteoarthritis", "Lipodistrophy AND joint health", "Leptin", 77 “Adiponectin”, "Visfatin”, “Resistin”, “Chemerin-1”, "Progranulin”, “Omentin”, “Lipocalin-2”, 78 "infapatellar fat pad", "intra-articular fat pad" and "Bone marrow adipose tissue AND lipids". 79 All articles identified were English-language papers. In addition relevant references from 80 selected publications and relevant references were identified. 


\section{Role of systemic adipose tissues}

\section{Description and physiology}

AT can be related to OA progression by biomechanical and metabolic mechanisms (Figure 1).

The biomechanical ones refer to an increase in body weight due to AT gain leading to abnormal loading on the joints. The metabolic ones include abnormal lipid profile and secretion of adipokines by adipocytes. Herein we summarize the implication of Subcutaneous (SCAT) and Visceral AT (VAT) on those mechanisms.

SCAT is situated beneath the skin whereas VAT fills the peritoneal cavity and the space between internal organs. Augmentation on either of them implies an increase on body weight and on joint loading. Mechanical stress is an important factor on OA initiation and development [1113]. Exercise produces a loss of AT weight which alleviates pain symptoms in OA patients. Regarding the metabolic component, SCAT explants from OA patients stimulated with IL1 $\beta$ have been reported to increase pro- and anti-inflammatory signals [14]. Visceral adipocytes seem to be more active in terms of lipolysis and lipogenesis and a major source of adipokines and cytokines in comparison to other types of adipocytes. Adipocytes are also found in the middle of skeletal muscles and their accumulation on females is correlated with OA progression $[11,15]$. Below we mention some of the most studied adipokines secreted by these different tissues and how they are related to OA. 


\section{Systemic adipokines and $\mathrm{OA}$}

104 Adipokines may play a role in early diagnosis and management of OA symptoms due to their 105 role on cartilage degradation, synovial inflammation and bone remodeling (Table). The 106 evaluation of adipokine content in clinical and experimental models is obtained from serum, 107 plasma or synovial fluid. Asides of AT, joint tissues participate in adipokines secretion. A great 108 amount of adipokines have been correlated to OA onset, development and progression, being 109 leptin the most studied one, followed by adiponectin, resistin and visfatin. Table summarizes 110 recently published data on adipokines, whereas the text below focuses on the best described 111 adipokines. These adipokines in OA drive pathways directly related to inflammation, cartilage 112 degradation, infiltration of joint tissues by immune cells, mesenchymal stem cell (MSCs) 113 differentiation, chondrocytes de-differentiation or osteoclast activation [16-18]. In addition, 114 resistin and visfatin have been described as markers of knee function while leptin and 115 adiponectin as pain markers in OA [19, 20], but further studies need to be performed.

116 Omentin-1 and vaspin have been reported to be secreted exclusively by VAT but their role 117 seems to be opposed to the rest of other adipokines. In vitro, they display chondro-protective 118 activity and are negatively related to OA severity [17*]. Leptin, adiponectin and visfatin could 119 also act under specific conditions as anti-inflammatory and anti-catabolic agents, avoiding tissue 120 degradation. Chemerin for instance could be a marker for obesity-associated OA and with a 121 possible role on innate immune system-associated inflammation on those patients, while 122 lipocalin-2 has been suggested to be a mechano-responsive adipokine [17*, 18]. Interestingly, 123 apelin is the only adipokine described so far to be directly involved with synovium angiogenesis, 124 a known marker of severity in OA [21]. Many other adipokines have been shown to have a 
125 possible role on OA $\left[17^{*}, 22\right]$. Researchers keep testing if those interesting molecules could serve on the early diagnosis of $\mathrm{OA}$ as well as targets for future therapeutic strategies.

\section{Role of intra-articular adipose tissues}

129

130

131

132

133

134

135

136

137

138

139

140

141

142

143

144

145

\section{Description and physiology}

Intra-articular adipose tissues (IAAT) are fat pads found between the synovium and the joint capsule. The best characterized and the largest IAAT is the infrapatellar fat pad (IFP). IAAT are white AT (WAT) as SCAT and VAT. Although their characteristics are close to those of VAT, IAAT share common features with SCAT that distinguish them from VAT [23*]. There is no clear consequence of high fat diet on adipocyte size or inflammation of IFP in mice, with contradictory published results [24-26]. Recent data on human OA patients reported an absence of link between obesity and IFP volume [27] or between BMI of OA patients and either adipocyte or inflammatory features of IFP [28], suggesting that IAAT may be different to SCAT and VAT and display specific functions.

The physiological roles of IAAT are still not well characterized. IFP was initially supposed by Clopton Harvers at the end of $17^{\text {th }}$ Century to secrete the synovial fluid and latter, by Jean Cruveilhier in the $19^{\text {th }}$ Century, to fill gaps in the joint. By increasing the synovial surface, IFP facilitates the distribution of the synovial fluid. It may protect the patellar tendon and the anterior horns of the menisci and may supply nutriments to the patellar ligament [29]. IFP is also supposed to act as a shock absorber during joint movement. More recently, it was shown that IFP secrete factors $[30,31]$, especially prostaglandin $F_{2 \alpha}$ and prostaglandin $E_{2}$, which induce a 
146 fibrotic and inflammatory response in fibroblast-like synoviocytes [32, 33], suggesting that 147 IAAT and synovium are partners of a same functional unit [23*, 34].

\section{IAAT and $O A$}

A debate exists for several years on the protective or detrimental effect of IAAT on OA. The 151 role of IFP as a shock absorber has been pointed out to explain its possible protective effect, as 152 recently reviewed $[11,35]$. A protective effect of IFP-secreted factors and IFP-derived MSCs 153 have been also proposed $[11,35]$. Nevertheless, meta-analyses showed little if any detrimental 154 effect of IFP resection on clinical outcomes after total knee arthroplasty [36-38]. On the other 155 side, alteration in magnetic resonance imaging signal intensity of IFP has been linked to OA 156 progression [39] and may predict both accelerated knee OA [40, 41] and knee replacement [42*].

157 Interestingly, with the aim of an early detection of OA progressors, Bonakdari et al. developed a 158 method to predict the volume of IFP [43]. Although the relationship between IFP volume and 159 OA remains unclear, IFP volume is related to patello-femoral joint OA pain [44]. IFP contains numerous sensitive fibers [45] and is considered as a major source of knee pain [46, 47]. OA

161 IAAT are characterized by inflammatory cell infiltration, fibrosis and increased vascularization $162\left[23^{*}, 48,49\right]$. Fibrosis and inflammation of IFP are known features of anterior knee pain. They 163 are associated with an increased vascularization and calcitonin-positive nerve fibers in the 164 fibrotic areas of IFP [50]. Similar observations were obtained with the monoiodoacetic acid 165 (MIA) model of OA, in which IFP changes occurred before cartilage degradation [51, 52].

166 IAAT secrete factors with proinflammatory and tissue remodeling activities [23*, 30, 31, 33, 167 49] (Figure 1). Interestingly, IFP from patients with OA and rheumatoid arthritis display distinct 168 fatty acid signatures [53], suggesting disease-specific phenotypes for IFP. The OA-specific 
secretory phenotype of IAAT may be directly involved in synovial inflammation and fibrosis $[23,32,33]$ since IFP remodeling precedes synovitis [52].

$$
\text { IAAT cellular composition comprises adipocytes, leukocytes, endothelial and mesenchymal }
$$
cells, all participating in the OA-specific secretory phenotype of IAAT $[49,54,55]$. Although the specific roles of IAAT macrophages remains unknown [56, 57], those of MSCs are more understood. Initially, an anti-inflammatory activity of IFP-derived MSCs from OA patients has been reported [58]. It has been recently proposed that IFP-derived MSCs may be deleterious in OA via their secretion of inflammatory factors, their ability to recruit monocytes and their exacerbated response to an inflammatory stimulus $[54,55]$. In addition, cell lineage tracing experiments identified IFP perivascular MSCs as able to transdifferentiate into myofibroblasts and induce IFP fibrosis in posttraumatic OA model [59, 60*]. Moreover, fibroblasts isolated from fibrotic IFP have been involved in inflammatory cell recruitment and pain [61*].

\section{Description and physiology}

\section{Role of bone marrow adipose tissue}

$$
\text { Bone marrow adipose tissue (BMAT) constitutes over 10\% of total adipose mass and 70\% of }
$$
the bone marrow (BM) volume in young lean healthy human adults. The initial concept of BMAT as a passive fat storage depot has been challenged in the recent years although little is known about its physiological roles. It is now well accepted that BMAT has a unique development, molecular profile, regulation and modulation of the anatomical context that make it different from the other types of AT. 
BMAT volume changes upon the pathophysiological conditions; it increases with ageing, obesity, type 2 diabetes, osteoporosis or skeletal unloading [62], whereas it decreases with exercise [63], mechanical loading and hormonal changes (Figure 2). BMAT can be classified into constitutive (cBMAT) and regulated BMAT (rBMAT). Both of them differ by the time of their development, their localization in the skeleton, their gene expression pattern and their content in saturated/unsatured lipids [64**]. These differences could indicate different functions and even different progenitors. Nevertheless, rBMAT could also change to a cBMAT phenotype under specific conditions [62].

BM adipocytes (BMAds) have one unilocular lipid droplet with abundant mitochondria [65] and their gene expression pattern is similar to white adipocytes [62]. It is believed that BMAds arise from BM MSCs, probably the same progenitors as osteoblasts. A recent study has proved the progenitors to be more white-like [66] even though it is possible multiple populations within the BMAds could exist [67]. BMAds secrete extracellular vesicles and numerous soluble factors, which may control bone microenvironment [62, 68**]. Zou et al. indeed recently showed that BMAds ablation provokes massive bone formation due to the activation of bone morphogenetic protein receptor signaling pathway in MSCs [69**]. In addition, lack of adipo-progenitors on mice produces bone loss and abnormal vasculature [70**].

Aside of its paracrine role, BMAT could regulate systemic metabolism. Moreover, patients with BMAT alteration frequently develop ectopic storage of fat resulting on insulin resistance [71]. BMAT lipogenesis is triggered by short-term cold exposure and is less dependent on insulin than WAT [66]. Little is known about the lipolysis mechanisms on BMAT, but it could be either cytoplasmic lipase-mediated or by lipophagy [68**, 72*]. Specifically, the uptake and esterification of fatty acids is greater in BMAT than in WAT and those fatty acids fuel 
213 hematopoietic tumors and their oxidation is crucial for hematopoietic stem cell maintenance [73,

214 74]. Suchacki et al. have shown that BMAds have high basal glucose uptake that is greater in the 215 axial skeleton than in long bones [66], suggesting that BMAT may influence systemic glucose 216 homeostasis and that this characteristic is needed to support normal metabolic function and de 217 novo lipogenesis.

\section{BMAT and $O A$}

Pathophysiological conditions where bone homeostasis is lost have been directly related to an increase in BMAT. Surprisingly, they all constitute OA risk factors. In addition, OA entails sub-

222 chondral bone remodeling and BM is the only tissue where adipocytes and bone cells are in close association. All of these argue for a possible role of BMAT on OA (Figure 1). Moreover, the

224 femoral heads from OA patients contained high amounts of fat and of $n-6$ fatty acids, especially 225 arachidonic acid [75] (Figure 3). Early this year, Collins et al. proposed that knee joints of

226 lipodystrophic mice were protected from spontaneous or post-traumatic OA, independently from 227 diet [76]. Susceptibility to post-traumatic OA was reintroduced using implantation of AT derived 228 from wild type animals, probably due to the paracrine signalling from fat [76]. Nevertheless, 229 lipodystrophic patients have multiple bone abnormalities such as subchondral bone sclerosis, 230 similar to OA patients [77]. Interestingly, osteoblasts and osteocytes can also accumulate lipids 231 [68]. The cross-talk between BMAT and joint tissues is far from being unveiled and more studies 232 are needed to describe the mechanisms involved on OA pathogenesis.

233 Since all joint tissues are of mesenchymal origin and OA is a whole joint disease, it is 234 possible that OA affects MSC features. Both the synthesis of a poorly mineralised matrix and 
235 high content of fat characterize OA bone. This may result from a defect on the differentiation 236 capacity of MSCs favouring preferentially adipogenic over osteogenic lineage. Moreover, a 237 direct role of sclerostin in inducing BM adipogenesis through inhibiting Wnt signaling has 238 recently been reported [78]. The inhibition of Wnt signaling increased expression of adipogenic 239 transcription factors Ppary and Cebp $\alpha$ and stimulated adipogenesis [79]. However, lack of 240 adiponectin-positive progenitors in mice leads to both bone and angiogenic defects [70].

241 The role of BMAT in OA still remains speculative but numerous arguments indicate that it 242 could be involved in the dysregulation of joint tissues in OA. Future studies are needed to 243 explore in detail the role of BMAT in OA.

\section{Conclusion}

246 The discovery of the role of low-grade inflammation in certain phenotypes of OA has opened 247 up new physiopathological hypotheses involving AT. The recent description of AT of various 248 phenotypes within an osteoarthritic joint allows us to evoke their direct involvement in the 249 initiation and progression of the osteoarthritic process (Figure 1). We can expect in the near 250 future the discovery of novel molecules targeting these tissues. 
- Visceral and subcutaneous adipose tissues secrete adipokines, which differentially affect joint

254 tissue homeostasis.

255

256

257 both accelerated knee osteoarthritis and replacement.

258

259

260

261

262

263

264

265

266

267

268

269

270

271

272

273

274 None. components of a same functional unit. osteoarthritis.

\section{Acknowledgments} SPF20160936284.

- Drafting of the article: NZL, FE, FB, XH

- Final approval of the article: NZL, FE, FB, XH

\section{Conflict of interest}

- Intra-articular adipose tissue fibrosis and inflammation are early events in osteoarthritis and alteration in magnetic resonance imaging signal intensity of infrapatellar fat pad may predict

- Inflammatory and remodeling factors secreted by intra-articular adipose tissue may be responsible for cell and tissue damages of both intra-articular adipose tissue and synovium, as

- Bone marrow adipose tissue is a newly studied adipose tissue and a known regulator of bone microenvironment. Its volume changes in pathophysiological conditions associated with osteoarthritis and its composition is enriched in $n-6$ fatty acids, especially arachidonic acid, in osteoarthritic patients, suggesting that it may be a new adipose tissue playing role in

This work was supported by a grant from the Société Française de Rhumatologie. Natalia Zapata-Linares was supported by a grant from the Fondation pour la Recherche Médicale:

\section{Author Contributions}




\section{References}

276 [1] Collaborators GDaH. Global, regional, and national disability-adjusted life-years (DALYs)

277 for 315 diseases and injuries and healthy life expectancy (HALE), 1990-2015: a systematic

278 analysis for the Global Burden of Disease Study 2015. Lancet 2016; 388:1603-1658.

279 [2] Felson DT, Anderson JJ, Naimark A et al. Obesity and knee osteoarthritis. The Framingham

280 Study. Ann Intern Med 1988; 109:18-24.

281 [3] Reijman M, Pols HA, Bergink AP et al. Body mass index associated with onset and 282 progression of osteoarthritis of the knee but not of the hip: the Rotterdam Study. Ann Rheum 283 Dis 2007; 66:158-62.

284 [4] Lohmander LS, Gerhardsson de Verdier M, Rollof J et al. Incidence of severe knee and hip 285 osteoarthritis in relation to different measures of body mass: a population-based prospective 286 cohort study. Ann Rheum Dis 2009; 68:490-6.

287 [5] Yusuf E, Nelissen RG, Ioan-Facsinay A et al. Association between weight or body mass 288 index and hand osteoarthritis: a systematic review. Ann Rheum Dis 2010; 69:761-5.

289 [6] Trayhurn P, Wood IS. Adipokines: inflammation and the pleiotropic role of white adipose 290 tissue. Br J Nutr 2004; 92:347-55.

291 [7] Matsubara M, Maruoka S, Katayose S. Inverse relationship between plasma adiponectin and 292 leptin concentrations in normal-weight and obese women. Eur J Endocrinol 2002; 147:17329380.

294 [8] de Boer TN, van Spil WE, Huisman AM et al. Serum adipokines in osteoarthritis; 295 comparison with controls and relationship with local parameters of synovial inflammation and 296 cartilage damage. Osteoarthritis Cartilage 2012; 20:846-53. 
297 [9] Gao YH, Zhao CW, Liu B et al. An update on the association between metabolic syndrome 298 and osteoarthritis and on the potential role of leptin in osteoarthritis. Cytokine 2020; $299 \quad 129: 155043$.

300 [10] Iannone F, Lapadula G. Obesity and inflammation--targets for OA therapy. Curr Drug $301 \quad$ Targets 2010; 11:586-98.

302 [11] Chang J, Liao Z, Lu M et al. Systemic and local adipose tissue in knee osteoarthritis. $303 \quad$ Osteoarthritis Cartilage 2018; 26:864-871.

304 [12] Ramage L, Nuki G, Salter DM. Signalling cascades in mechanotransduction: cell-matrix 305 interactions and mechanical loading. Scand J Med Sci Sports 2009; 19:457-69.

[13] Visser AW, de Mutsert R, le Cessie S et al. The relative contribution of mechanical stress and systemic processes in different types of osteoarthritis: the NEO study. Ann Rheum Dis $2015 ; 74: 1842-7$

[14] Kontny E, Zielinska A, Ksiezopolska-Orlowska K, Gluszko P. Secretory activity of subcutaneous abdominal adipose tissue in male patients with rheumatoid arthritis and osteoarthritis - association with clinical and laboratory data. Reumatologia 2016; 54:227-235.

[15] Li S, Schwartz AV, LaValley MP et al. Association of Visceral Adiposity With Pain but Not Structural Osteoarthritis. Arthritis Rheumatol 2020; 72:1103-1110.

$316 *[17] \mathrm{Tu} \mathrm{C}, \mathrm{He} \mathrm{J}, \mathrm{Wu} \mathrm{B}$ et al. An extensive review regarding the adipokines in the pathogenesis 317 and progression of osteoarthritis. Cytokine 2019; 113:1-12.

318 A detailed review on the different cytokines with a role on OA pathogenesis. 
319 [18] Xie C, Chen Q. Adipokines: New Therapeutic Target for Osteoarthritis? Curr Rheumatol $320 \quad \operatorname{Rep} 2019 ; 21: 71$.

321 [19] Calvet J, Orellana C, Albinana Gimenez N et al. Differential involvement of synovial 322 adipokines in pain and physical function in female patients with knee osteoarthritis. A cross323 sectional study. Osteoarthritis Cartilage 2018; 26:276-284.

324 [20] Askari A, Arasteh P, Homayounfar R et al. The role of adipose tissue secretion in the 325 creation and pain level in osteoarthritis. Endocr Regul 2020; 54:6-13.

326 [21] Wang YH, Kuo SJ, Liu SC et al. Apelin Affects the Progression of Osteoarthritis by 327 Regulating VEGF-Dependent Angiogenesis and miR-150-5p Expression in Human Synovial $328 \quad$ Fibroblasts. Cells 2020; 9.

329 [22] Scotece M, Koskinen-Kolasa A, Pemmari A et al. Novel adipokine associated with OA: 330 retinol binding protein $4(\mathrm{RBP} 4)$ is produced by cartilage and is correlated with MMPs in $331 \quad$ osteoarthritis patients. Inflamm Res 2020; 69:415-421.

$332 *[23]$ Eymard F, Pigenet A, Citadelle D et al. Knee and hip intra-articular adipose tissues 333 (IAATs) compared with autologous subcutaneous adipose tissue: a specific phenotype for a 334 central player in osteoarthritis. Ann Rheum Dis 2017; 76:1142-1148.

335 An interesting study showing that IAAT from knee and hip of OA patients share similar 336 charecteristics that differ from those of SCAT but also from VAT. This study defines IAAT and 337 synovium as a same functional unit.

338 [24] Iwata M, Ochi H, Hara Y et al. Initial responses of articular tissues in a murine high-fat 339 diet-induced osteoarthritis model: pivotal role of the IPFP as a cytokine fountain. PLoS One $340 \quad 2013 ; 8: e 60706$. 
[25] Barboza E, Hudson J, Chang WP et al. Profibrotic Infrapatellar Fat Pad Remodeling Without M1 Macrophage Polarization Precedes Knee Osteoarthritis in Mice With DietInduced Obesity. Arthritis Rheumatol 2017; 69:1221-1232.

[26] Warmink K, Kozijn AE, Bobeldijk I et al. High-fat feeding primes the mouse knee joint to develop osteoarthritis and pathologic infrapatellar fat pad changes after surgically induced injury. Osteoarthritis Cartilage 2020; 28:593-602.

[27] Masaki T, Takahashi K, Hashimoto S et al. Volume change in infrapatellar fat pad is associated not with obesity but with cartilage degeneration. J Orthop Res 2019; 37:593-600.

[28] de Jong AJ, Klein-Wieringa IR, Andersen SN et al. Lack of high BMI-related features in adipocytes and inflammatory cells in the infrapatellar fat pad (IFP). Arthritis Res Ther 2017; 19:186.

[29] Eymard F, Chevalier X. Inflammation of the infrapatellar fat pad. Joint Bone Spine 2016; 83:389-93.

[30] Distel E, Cadoudal T, Durant S et al. The infrapatellar fat pad in knee osteoarthritis: an important source of interleukin-6 and its soluble receptor. Arthritis Rheum 2009; 60:3374-7.

[31] Ushiyama T, Chano T, Inoue K, Matsusue Y. Cytokine production in the infrapatellar fat pad: another source of cytokines in knee synovial fluids. Ann Rheum Dis 2003; 62:108-12.

[32] Bastiaansen-Jenniskens YM, Wei W, Feijt C et al. Stimulation of fibrotic processes by the infrapatellar fat pad in cultured synoviocytes from patients with osteoarthritis: a possible role for prostaglandin f2alpha. Arthritis Rheum 2013; 65:2070-80.

[33] Eymard F, Pigenet A, Citadelle D et al. Induction of an inflammatory and prodegradative phenotype in autologous fibroblast-like synoviocytes by the infrapatellar fat pad from patients with knee osteoarthritis. Arthritis Rheumatol 2014; 66:2165-74. 
364 [34] Macchi V, Stocco E, Stecco C et al. The infrapatellar fat pad and the synovial membrane: an anatomo-functional unit. J Anat 2018; 233:146-154.

366 [35] Jiang LF, Fang JH, Wu LD. Role of infrapatellar fat pad in pathological process of knee osteoarthritis: Future applications in treatment. World J Clin Cases 2019; 7:2134-2142.

[36] Sun C, Zhang X, Lee WG et al. Infrapatellar fat pad resection or preservation during total knee arthroplasty: a meta-analysis of randomized controlled trials. J Orthop Surg Res 2020; $15: 297$.

371 [37] White L, Holyoak R, Sant J et al. The effect of infrapatellar fat pad resection on outcomes post-total knee arthroplasty: a systematic review. Arch Orthop Trauma Surg 2016; 136:701-8.

[38] Ye C, Zhang W, Wu W et al. Influence of the Infrapatellar Fat Pad Resection during Total $374 \quad$ Knee Arthroplasty: A Systematic Review and Meta-Analysis. PLoS One 2016; 11:e0163515.

375 [39] Ruhdorfer A, Haniel F, Petersohn T et al. Between-group differences in infra-patellar fat 376 pad size and signal in symptomatic and radiographic progression of knee osteoarthritis vs 377 non-progressive controls and healthy knees - data from the FNIH Biomarkers Consortium 378 Study and the Osteoarthritis Initiative. Osteoarthritis Cartilage 2017; 25:1114-1121.

379 [40] Davis JE, Ward RJ, MacKay JW et al. Effusion-synovitis and infrapatellar fat pad signal 380 intensity alteration differentiate accelerated knee osteoarthritis. Rheumatology (Oxford) 2019; $381 \quad 58: 418-426$.

382 [41] Harkey MS, Davis JE, Lu B et al. Early pre-radiographic structural pathology precedes the 383 onset of accelerated knee osteoarthritis. BMC Musculoskelet Disord 2019; 20:241.

$384 *[42]$ Wang K, Ding C, Hannon MJ et al. Signal intensity alteration within infrapatellar fat pad 385 predicts knee replacement within 5 years: data from the Osteoarthritis Initiative. Osteoarthritis $386 \quad$ Cartilage 2018; 26:1345-1350. 
A clinical study showing that IFP alterations revealed by MRI predicts knee replacement within 5 years.

[43] Bonakdari H, Tardif G, Abram F et al. Serum adipokines/related inflammatory factors and ratios as predictors of infrapatellar fat pad volume in osteoarthritis: Applying comprehensive machine learning approaches. Sci Rep 2020; 10:9993.

[44] Cowan SM, Hart HF, Warden SJ, Crossley KM. Infrapatellar fat pad volume is greater in individuals with patellofemoral joint osteoarthritis and associated with pain. Rheumatol Int $2015 ; 35: 1439-42$.

[45] Bohnsack M, Meier F, Walter GF et al. Distribution of substance-P nerves inside the infrapatellar fat pad and the adjacent synovial tissue: a neurohistological approach to anterior knee pain syndrome. Arch Orthop Trauma Surg 2005; 125:592-7.

[46] Belluzzi E, Stocco E, Pozzuoli A et al. Contribution of Infrapatellar Fat Pad and Synovial Membrane to Knee Osteoarthritis Pain. Biomed Res Int 2019; 2019:6390182.

[47] Draghi F, Ferrozzi G, Urciuoli L et al. Hoffa's fat pad abnormalities, knee pain and magnetic resonance imaging in daily practice. Insights Imaging 2016; 7:373-83.

[48] Favero M, El-Hadi H, Belluzzi E et al. Infrapatellar fat pad features in osteoarthritis: a histopathological and molecular study. Rheumatology (Oxford) 2017; 56:1784-1793.

[49] Klein-Wieringa IR, Kloppenburg M, Bastiaansen-Jenniskens YM et al. The infrapatellar fat pad of patients with osteoarthritis has an inflammatory phenotype. Ann Rheum Dis 2011; 70:851-7.

[50] Onuma H, Tsuji K, Hoshino T et al. Fibrotic changes in the infrapatellar fat pad induce new vessel formation and sensory nerve fiber endings that associate prolonged pain. J Orthop Res 2020; 38:1296-1306. 
410 [51] Clements KM, Ball AD, Jones $\mathrm{HB}$ et al. Cellular and histopathological changes in the 411 infrapatellar fat pad in the monoiodoacetate model of osteoarthritis pain. Osteoarthritis $412 \quad$ Cartilage 2009; 17:805-12.

413 [52] Inomata $\mathrm{K}$, Tsuji $\mathrm{K}$, Onuma $\mathrm{H}$ et al. Time course analyses of structural changes in the 414 infrapatellar fat pad and synovial membrane during inflammation-induced persistent pain 415 development in rat knee joint. BMC Musculoskelet Disord 2019; 20:8.

416 [53] Mustonen AM, Kakela R, Lehenkari P et al. Distinct fatty acid signatures in infrapatellar fat 417 pad and synovial fluid of patients with osteoarthritis versus rheumatoid arthritis. Arthritis Res $418 \quad$ Ther $2019 ; 21: 124$.

419 [54] Bravo B, Guisasola MC, Vaquero J et al. Gene expression, protein profiling, and 420 chemotactic activity of infrapatellar fat pad mesenchymal stem cells in pathologies of the $421 \quad$ knee joint. J Cell Physiol 2019; 234:18917-18927.

422 [55] Eymard F, Pigenet A, Rose C et al. Contribution of adipocyte precursors in the phenotypic 423 specificity of intra-articular adipose tissues in knee osteoarthritis patients. Arthritis Res Ther $424 \quad 2019 ; 21: 252$.

425 [56] Wu CL, Harasymowicz NS, Klimak MA et al. The role of macrophages in osteoarthritis and 426 cartilage repair. Osteoarthritis Cartilage 2020; 28:544-554.

427 [57] Xie J, Huang Z, Yu X et al. Clinical implications of macrophage dysfunction in the 428 development of osteoarthritis of the knee. Cytokine Growth Factor Rev 2019; 46:36-44.

429 [58] Manferdini C, Maumus M, Gabusi E et al. Adipose-derived mesenchymal stem cells exert 430 antiinflammatory effects on chondrocytes and synoviocytes from osteoarthritis patients 431 through prostaglandin E2. Arthritis Rheum 2013; 65:1271-81. 
432 [59] Sono T, Hsu CY, Negri S et al. Platelet-derived growth factor receptor-beta (PDGFRbeta)

433 lineage tracing highlights perivascular cell to myofibroblast transdifferentiation during post-

434 traumatic osteoarthritis. J Orthop Res 2020.

$435 *[60]$ Sono T, Hsu CY, Wang Y et al. Perivascular Fibro-Adipogenic Progenitor Tracing during

436 Post-Traumatic Osteoarthritis. Am J Pathol 2020; 190:1909-1920.

437 Using cell lineage tracing, this study reveals the importance of perivascular MSC in IFP 438 fibrosis.

$439 *$ *61] Paish HL, Kalson NS, Smith GR et al. Fibroblasts Promote Inflammation and Pain via IL440 1alpha Induction of the Monocyte Chemoattractant Chemokine (C-C Motif) Ligand 2. Am J $441 \quad$ Pathol 2018; 188:696-714.

442 An interesting study that highlights the role of fibrosis and fibroblasts in IFP inflammation and 443 pain.

444 [62] Li Y, Meng Y, Yu X. The Unique Metabolic Characteristics of Bone Marrow Adipose $445 \quad$ Tissue. Front Endocrinol (Lausanne) 2019; 10:69.

446 [63] Patel VS, Ete Chan M, Rubin J, Rubin CT. Marrow Adiposity and Hematopoiesis in Aging 447 and Obesity: Exercise as an Intervention. Curr Osteoporos Rep 2018; 16:105-115.

$448 * *[64]$ Scheller EL, Doucette CR, Learman BS et al. Region-specific variation in the properties 449 of skeletal adipocytes reveals regulated and constitutive marrow adipose tissues. Nat $450 \quad$ Commun 2015; 6:7808.

451 One of the first studies focused on the molecular characterization of BMAT. Notably, this study 452 evidences the existence of several subtypes of BMAT with different behaviors.

453 [65] Li Z, Hardij J, Bagchi DP et al. Development, regulation, metabolism and function of bone $454 \quad$ marrow adipose tissues. Bone 2018; 110:134-140. 
455 [66] Suchacki KJ, Tavares AAS, Mattiucci D et al. Bone marrow adipose tissue is a unique 456 adipose subtype with distinct roles in glucose homeostasis. Nat Commun 2020; 11:3097.

457 [67] Horowitz MC, Berry R, Holtrup B et al. Bone marrow adipocytes. Adipocyte 2017; 6:193458204.

$459 * *[68]$ Rendina-Ruedy E, Rosen CJ. Lipids in the Bone Marrow: An Evolving Perspective. Cell $460 \quad$ Metab 2020; 31:219-231.

461 A detailed review specific on the lipid content of bone marrow adipose tissue that could help to $462 \quad$ bring some light on the still unknown functions of this tissue.

$463 * *[69]$ Zou W, Rohatgi N, Brestoff JR et al. Ablation of Fat Cells in Adult Mice Induces $464 \quad$ Massive Bone Gain. Cell Metab 2020.

465 An interesting study showing that BMADs display a negative control on bone mass via the 466 secretion of inhibitors of bone morphogenetic protein receptor signaling pathway in MSCs.

$467 * *[70]$ Zhong L, Yao L, Tower RJ et al. Single cell transcriptomics identifies a unique adipose $468 \quad$ lineage cell population that regulates bone marrow environment. Elife 2020; 9.

469 An original article revealing a progenitor population for bone marrow adipocytes and showing $470 \quad$ finally the need to find a balance between adipogenesis and bone remodeling.

471 [71] Yamamoto A, Kusakabe T, Sato K et al. Seipin-linked congenital generalized lipodystrophy 472 type 2: a rare case with multiple lytic and pseudo-osteopoikilosis lesions. Acta Radiol Open $473 \quad 2019 ; 8: 2058460119892407$.

$474 *$ [72] Sebo ZL, Rendina-Ruedy E, Ables GP et al. Bone Marrow Adiposity: Basic and Clinical 475 Implications. Endocr Rev 2019; 40:1187-1206.

476 A Review on the bone marrow adipocyte characteristics in comparison to other adipose tissues. 
477 [73] Diedrich JD, Herroon MK, Rajagurubandara E, Podgorski I. The Lipid Side of Bone 478 Marrow Adipocytes: How Tumor Cells Adapt and Survive in Bone. Curr Osteoporos Rep $479 \quad 2018 ; 16: 443-457$.

480 [74] Zhang Z, Huang Z, Ong B et al. Bone marrow adipose tissue-derived stem cell factor 481 mediates metabolic regulation of hematopoiesis. Haematologica 2019; 104:1731-1743.

482 [75] Plumb MS, Aspden RM. High levels of fat and (n-6) fatty acids in cancellous bone in $483 \quad$ osteoarthritis. Lipids Health Dis 2004; 3:12.

484 [76] Collins KH, Lenz KL, Pollitt EN et al. Adipose Tissue is a Critical Regulator of 485 Osteoarthritis. bioRxiv 2020; doi: 10.1101/2020.06.04.134601.

486 [77] Teboul-Core S, Rey-Jouvin C, Miquel A et al. Bone imaging findings in genetic and 487 acquired lipodystrophic syndromes: an imaging study of 24 cases. Skeletal Radiol 2016; $488 \quad 45: 1495-506$.

489 [78] Lories RJ, Monteagudo S. Review Article: Is Wnt Signaling an Attractive Target for the $490 \quad$ Treatment of Osteoarthritis? Rheumatol Ther 2020; 7:259-270.

491 [79] Fairfield H, Falank C, Harris E et al. The skeletal cell-derived molecule sclerostin drives 492 bone marrow adipogenesis. J Cell Physiol 2018; 233:1156-1167.

493 [80] Boffa A, Merli G, Andriolo L et al. Synovial Fluid Biomarkers in Knee Osteoarthritis: A 494 Systematic Review and Quantitative Evaluation Using BIPEDs Criteria. Cartilage $495 \quad 2020: 1947603520942941$.

496 [81] Sachdeva M, Aggarwal A, Sharma R et al. Chronic inflammation during osteoarthritis is 497 associated with an increased expression of CD161 during advanced stage. Scand J Immunol $498 \quad 2019 ; 90: \mathrm{e} 12770$. 
499 [82] Min S, Shi T, Han X et al. Serum levels of leptin, osteopontin, and sclerostin in patients $500 \quad$ with and without knee osteoarthritis. Clin Rheumatol 2020.

501 [83] Yan M, Zhang J, Yang H, Sun Y. The role of leptin in osteoarthritis. Medicine (Baltimore) $502 \quad 2018 ; 97: \mathrm{e} 0257$.

503 [84] Xiao K, Yu L, Zhu L et al. Urine Proteomics Profiling and Functional Characterization of $504 \quad$ Knee Osteoarthritis Using iTRAQ Technology. Horm Metab Res 2019; 51:735-740.

505 [85] Shang H, Hao Y, Hu W et al. Association between ADIPOQ gene variants and knee $506 \quad$ osteoarthritis in a Chinese population. Biosci Rep 2019; 39.

507 [86] Cheleschi S, Gallo I, Barbarino M et al. MicroRNA Mediate Visfatin and Resistin Induction 508 of Oxidative Stress in Human Osteoarthritic Synovial Fibroblasts Via NF-kappaB Pathway. $509 \quad$ Int J Mol Sci 2019; 20.

510 [87] Yapici Yavuz G, Simsek Kaya G, Kiziltunc A. Analysis of synovial fluid visfatin level in 511 temporomandibular joint disorders. Cranio 2019; 37:296-303.

512 [88] Cheleschi S, Tenti S, Mondanelli N et al. MicroRNA-34a and MicroRNA-181a Mediate 513 Visfatin-Induced Apoptosis and Oxidative Stress via NF-kappaB Pathway in Human $514 \quad$ Osteoarthritic Chondrocytes. Cells 2019; 8.

515 [89] Macfadyen MA, Daniel Z, Kelly S et al. The commercial pig as a model of spontaneously$516 \quad$ occurring osteoarthritis. BMC Musculoskelet Disord 2019; 20:70.

517 [90] Alissa EM, Alzughaibi LS, Marzouki ZM. Relationship between serum resistin, body fat 518 and inflammatory markers in females with clinical knee osteoarthritis. Knee 2020; 27:45-50.

519 [91] Chen WC, Lin CY, Kuo SJ et al. Resistin Enhances VCAM-1 Expression and Monocyte 520 Adhesion in Human Osteoarthritis Synovial Fibroblasts by Inhibiting MiR-381 Expression 521 through the PKC, p38, and JNK Signaling Pathways. Cells 2020; 9. 
522 [92] Cajas Santana LJ, Rondon Herrera F, Rojas AP et al. Serum chemerin in a cohort of 523 Colombian patients with primary osteoarthritis. Reumatol Clin 2020.

524 [93] Pirozzi C, Francisco V, Guida FD et al. Butyrate Modulates Inflammation in Chondrocytes 525 via GPR43 Receptor. Cell Physiol Biochem 2018; 51:228-243.

526 [94] Feng D, Kang X, Wang R et al. Progranulin modulates cartilage-specific gene expression 527 via sirtuin 1-mediated deacetylation of the transcription factors SOX9 and P65. J Biol Chem 528

529 [95] Zhi L, Zhao J, Zhao H et al. Downregulation of LncRNA OIP5-AS1 Induced by IL-1beta 530 Aggravates Osteoarthritis via Regulating miR-29b-3p/PGRN. Cartilage 2020:1947603519900801.

[96] Jiang L, Xu K, Li J et al. Nesfatin-1 suppresses interleukin-1beta-induced inflammation, apoptosis, and cartilage matrix destruction in chondrocytes and ameliorates osteoarthritis in rats. Aging (Albany NY) 2020; 12:1760-1777.

[97] Wang Q, Xu X, Kang Z et al. Paeonol prevents IL-1beta-induced inflammatory response 536 and degradation of type II collagen in human primary chondrocytes. Artif Cells Nanomed

538 [98] Conde J, Scotece M, Abella V et al. Identification of novel adipokines in the joint. 539 Differential expression in healthy and osteoarthritis tissues. PLoS One 2015; 10:e0123601.

540 [99] Li H, Yang HH, Sun ZG et al. Whole-transcriptome sequencing of knee joint cartilage from $541 \quad$ osteoarthritis patients. Bone Joint Res 2019; 8:288-301.

542 [100] Sanchez C, Mazzucchelli G, Lambert C et al. Comparison of secretome from osteoblasts 543 derived from sclerotic versus non-sclerotic subchondral bone in OA: A pilot study. PLoS One $544 \quad 2018 ; 13: \mathrm{e} 0194591$. 
545 [101] Tang S, Deng S, Guo J et al. Deep Coverage Tissue and Cellular Proteomics Revealed IL-

546 1beta Can Independently Induce the Secretion of TNF-Associated Proteins from Human

547 Synoviocytes. J Immunol 2018; 200:821-833.

548

549 
Table. Adipokine in osteoarthritis

\begin{tabular}{|c|c|c|c|}
\hline Adipokine & Source of detection & Described action & References 2018-2020 \\
\hline \multirow{3}{*}{ Leptin } & Plasma & Synovial fluid human knee OA biomarker & {$[80,81]$} \\
\hline & Serum & $\begin{array}{l}\text { Remarkable diagnostic value in the incidence of human } \\
\text { knee OA }\end{array}$ & [82] \\
\hline & Synovial Fluid & $\begin{array}{l}\text { Leptin and its receptor may be an emerging target for } \\
\text { intervention in human metabolic-associated OA. }\end{array}$ & {$[9,83]$} \\
\hline \multirow{3}{*}{ Adiponectin } & \multirow{3}{*}{$\begin{array}{l}\text { Serum } \\
\text { Synovial Fluid }\end{array}$} & Promising biomarker on human OA pathogenesis & {$[84]$} \\
\hline & & $\begin{array}{l}\text { Low levels observed in synovial fluids patients of lower OA } \\
\text { grades. }\end{array}$ & [81] \\
\hline & & Gene polymorphism intensifies the risk of human knee $O A$ & [85] \\
\hline \multirow{3}{*}{ Visfatin } & \multirow{3}{*}{ Synovial fluid } & Oxidative stress induction human in OA Synoviocytes & {$[86,87]$} \\
\hline & & $\begin{array}{l}\text { Human cartilage catabolic effects (Apoptosis, matrix } \\
\text { degradation, oxidative stress) }\end{array}$ & [88] \\
\hline & & Bone remodeling on pig OA model & [89] \\
\hline \multirow{4}{*}{ Resistin } & \multirow{4}{*}{$\begin{array}{l}\text { Plasma } \\
\text { Serum } \\
\text { Synovial Fluid }\end{array}$} & Modulates OA miRs with Visfatin & [86] \\
\hline & & Progression and pathogenesis of human knee OA & \multirow{2}{*}{ [90] } \\
\hline & & Novel and reliable biomarker for human OA severity & \\
\hline & & Pro-inflammatory effects in human OA & [91] \\
\hline
\end{tabular}




\begin{tabular}{|c|c|c|c|}
\hline Adipokine & Source of detection & Described action & References 2018-2020 \\
\hline \multirow{3}{*}{ Chemerin } & \multirow{3}{*}{ Serum } & Cartilage degradation & \multirow{3}{*}[92]{} \\
\hline & & Inflammation & \\
\hline & & $\begin{array}{l}\text { Found on serums of patients with primary OA of the hand, } \\
\text { knee or hip }\end{array}$ & \\
\hline Omentin-1 & $\begin{array}{l}\text { Synovial Fluid } \\
\text { Serum }\end{array}$ & Possible chondroprotective role in human cells & {$[16]$} \\
\hline \multirow[t]{2}{*}{ Vaspin } & \multirow[t]{2}{*}{ In vitro } & Possible anti-catabolic effect in human cartilage & \multirow[t]{2}{*}[16]{} \\
\hline & & Possible anti-inflammatory effect & \\
\hline \multirow[b]{2}{*}{ Lipocalin-2 } & \multirow[b]{2}{*}{ Synovial Fluid } & Pro-inflammatory effects in human OA & \multirow[b]{2}{*}{ [93] } \\
\hline & & $\begin{array}{l}\text { Its downregulation reduces chondrocyte inflammation } \\
\text { and cartilage degradation }\end{array}$ & \\
\hline \multirow{2}{*}{ Apelin } & \multirow{2}{*}{ In vitro human cells } & Angiogenesis synovium & \multirow{2}{*}[21]{} \\
\hline & & Catabolic effects & \\
\hline \multirow[b]{2}{*}{ Progranulin } & \multirow[b]{2}{*}{ In vitro human cells } & Triggers anabolic markers & \multirow[b]{2}{*}[94,95]{} \\
\hline & & Anti-inflammatory and anti-catabolic effects & \\
\hline \multirow{2}{*}{ Nesfatin-1 } & \multirow{2}{*}{$\begin{array}{l}\text { In vitro human cells } \\
\text { Animal model }\end{array}$} & Possible protective role in the development of OA & {$[96]$} \\
\hline & & Upregulated in OA chondrocytes & [97] \\
\hline
\end{tabular}




\begin{tabular}{|c|c|c|c|}
\hline Adipokine & Source of detection & Described action & References 2018-2020 \\
\hline \multirow{2}{*}{ RBP4 } & & Matrix degradation in human cartilage & \multirow{2}{*}{ [22] } \\
\hline & Blood samples & Positive correlation with other $\mathrm{OA}$ adipokines & \\
\hline $\begin{array}{l}\text { New Adipokines } \\
\text { (SERPINE2, } \\
\text { WISP2, GPBMB, } \\
\text { ITIH5) }\end{array}$ & In vitro & $\begin{array}{l}\text { Secreted by human OA chondrocytes, human OA sclerotic } \\
\text { subchondral bone, human OA synovial tissues and human } \\
\text { OA IAAT }\end{array}$ & {$[98-101]$} \\
\hline
\end{tabular}




\section{Figure legend}

Figure 1. Roles of the different adipose tissues on OA progression by biomechanical and metabolic mechanisms.

Increases on systemic AT like SCAT (Subcutaneous adipose tissue), VAT (Visceral adipose tissue) and intra-muscular adipose tissue contribute to abnormal loading of the joint, this mechanical stress have been shown to be part of OA onset and progression. Lipocalin adipokine family has emerged as sensors of mechanical load, inflammatory status and catabolic stimuli of the joint, suggesting its involvement in OA pathophysiology. On the other hand, the paracrine role of SCAT, VAT, intra-muscular AT and local AT BMAT (Bone marrow adipose tissue) and IAAT (intra-articular adipose tissue) affect joint health. The adipokines secreted by all those tissues have proven to promote directly: 1 . Secretion of inflammatory cytokines like Interleukin1beta (IL-1 $\beta$ ) and tumor necrosis factor-alpha (TNF- $\alpha$ ) which are well-documented for their active involvement in the pathophysiology of OA, 2. Cartilage catabolism, including inhibition of proliferation in chondrocytes and degradation of the matrix components, collagen type 2 and agrecan, 3. Immune response by the infiltration of joint tissues by monocytes and leucocytes which increases even more the inflmmatory signals present on the joint affected, 4. Loss of balance between osteoclast and osteoblast affecting directly bone remodeling, changes on bone constitution are part of OA pathology and 5. Changes on stem cell principal characteristics like prolifereation and differentiation capacity. 


\section{Figure 2. General characteristics of bone marrow adipose tissue.}

BMAT is currently considered as a tissue with significant paracrine and endocrine activities which make it a major player on different pathologies. BM adipocytes' gene expression pattern is similar to white-like adipocytes, they have one unilocular lipid droplet with abundant mitochondria and recent study has proved the progenitors to be more white-like. Their secretory profile includes extracellular vesicles and numerous molecules like inflammatory factors, adipokines or RANKL. BMAT is a unique adipose tissue which functions are still to be reveled. BMAT has a high intrinsic plasticity, increases with age as well as in other pathological contexts like: obesity, type 2 diabetes or osteoporosis. BMAT content can also decrease with exercise, mechanical loading or hormonal changes. In terms of development it can be classified into cBMAT or constitutive BMAT and rBMAT or regulated BMAT. cBMAT developed early in life, located in the distal skeleton, repository of unsaturated lipids and constituted by adipocytes larger in size with reduced expression of adipogenic markers. On the other hand, rBMAT increases with age, is located in the proximal skeleton where the adipocytes contain saturated lipids and express high levels of known adipogenic markers.

Figure 3. Possible role of bone marrow adiposity in joint health.

BMAT may play a role on inflammation, subchondral bone sclerosis, aberrant angiogenesis, adipogenic differentiation and bone remodeling all of them involved on joint health and OA development and progression. Femoral heads from OA patients contain high amounts of fat, especially arachidonic acid precursor of prostaglandin $E_{2}$ a known participant on OA inflammation [75]. Lipodystrophic mice were protected from spontaneous or post-traumatic OA, 
this study proposes that adipose tissue is a critical antagonist of cartilage health and integrity due precisely to the paracrine signalling from fat [76]. Mice without adiponectin-positive progenitors had elevated trabecular bone mass and their vessels within the bone marrow were less in number and high in diameter; characteristics that were far from normal. Sclerostin produced by the bonemechanosensing osteocytes inhibits Wnt signaling stimulated adipogenesis of mouse MSCs and human MSCs [79]. Nevertheless, the cross-talk between all joint tissues and BMAT is far from being unveiled and more studies are needed to describe the mechanisms, adipokines, pathways and signalling involved on OA pathogenesis. OA BMAd (bone marrow adipocytes from OA patients), Pre-BMAd (bone marrow adipocytes precursors). 


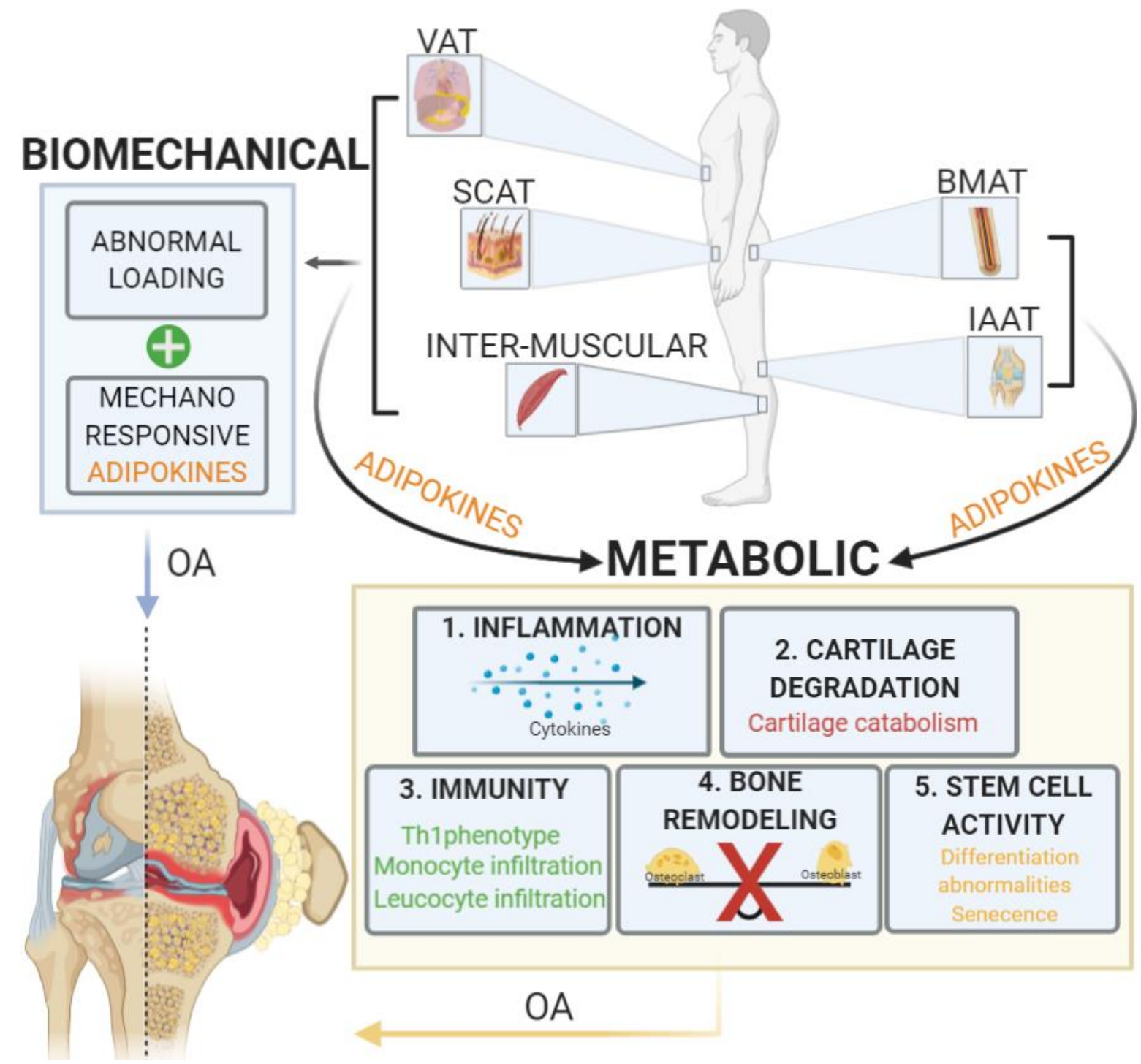

Figure 1 


\section{BONE MARROW ADIPOSE TISSUE}

BM adipocytes
Unilocular lipid droplet
Abundant mitochondria
Arise from BM MSCs
White-like genes

\section{Secretion}

Extracellular vesicles

Adipokines

Inflammatory factors

RANKL
Regulation

Up:

ageing

obesity

type 2 diabetes

osteoporosis

skeletal unloading

Down:

exercise

mechanical loading

hormonal changes

\section{Classification}

IBMAT:

proximal/central skeletal regions

develops later

source of saturated lipids

CBMAT:

distal/caudal skeletal regions

develops early in life

source of unsaturated lipids

larger adipocytes

Figure 2 


\section{BONE MARROW ADIPOSITY AND JOINT HEALTH}

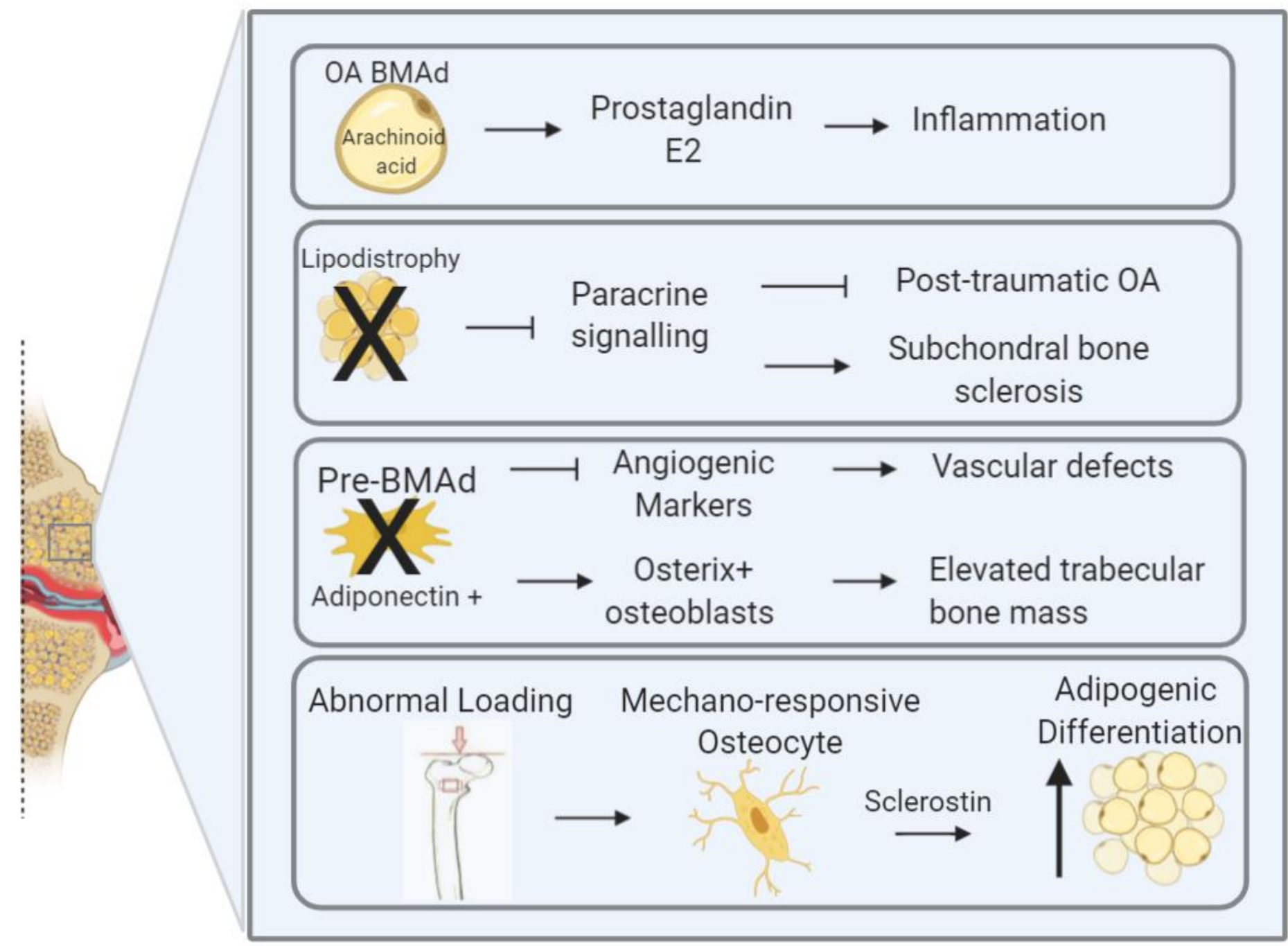

Figure 3 